PROCEEDINGS OF THE

AMERICAN MATHEMATICAL SOCIETY

Volume 125, Number 12, December 1997, Pages 3655-3658

S 0002-9939(97)04057-4

\title{
CHARACTERIZATION OF CHAOTIC ORDER AND ITS APPLICATION TO FURUTA INEQUALITY
}

\author{
MASATOSHI FUJII, JIAN FEI JIANG, AND EIZABURO KAMEI \\ (Communicated by Palle E. T. Jorgensen) \\ Dedicated to Professor P. R. Halmos on his 80th Birthday
}

\begin{abstract}
In this note, we give a simple characterization of the chaotic order $\log A \geq \log B$ among positive invertible operators $A, B$ on a Hilbert space. As an application, we discuss Furuta's type operator inequality.
\end{abstract}

\section{INTRODUCTION}

A (bounded linear) operator $A$ on a Hilbert space $H$ is positive, in symbols $A \geq 0$, if $(A x, x) \geq 0$ for all $x \in H$. And $A>0$ means that $A$ is positive invertible. It is well-known that $A \geq B \geq 0$ does not assure $A^{2} \geq B^{2}$ in general, but the LöwnerHeinz inequality says that the function $t \rightarrow t^{\alpha}$ on $[0, \infty)$ is operator monotone for $0 \leq \alpha \leq 1$, i.e.,

$$
A \geq B \geq 0 \text { implies } A^{\alpha} \geq B^{\alpha},
$$

cf. [8]. Furuta [5] gave it an ingenious extension which is called the Furuta inequality (cf. [2], [7] and [6] for an elementary and one-page proof):

If $A \geq B \geq 0$, then

$$
\left(A^{r} A^{p} A^{r}\right)^{1 / q} \geq\left(A^{r} B^{p} A^{r}\right)^{1 / q}
$$

and

$$
\left(B^{r} A^{p} B^{r}\right)^{1 / q} \geq\left(B^{r} B^{p} B^{r}\right)^{1 / q}
$$

holds for $r \geq 0, p \geq 0$ and $q \geq 1$ with $(1+2 r) q \geq p+2 r$ (see Figure 1).

Since $\log t$ is operator monotone, i.e., $\log A \geq \log B$ for $A \geq B>0$, it induces a weaker order $\gg$ among positive invertible operators than the usual one $\geq$, which is called the chaotic order, cf. [3]. Now Ando's theorem [1] is rephrased as a characterization of the chaotic order via a form of (2): For $A, B>0, A \gg B$ if and only if

$$
\left(A^{\frac{p}{2}} B^{p} A^{\frac{p}{2}}\right)^{\frac{1}{2}} \leq A^{p}
$$

holds for all $p \geq 0$.

Afterwards, it is extended to the following result [3], cf. [4].

Received by the editors July 16, 1996.

1991 Mathematics Subject Classification. Primary 47A63, 47B15.

Key words and phrases. Positive operators, chaotic order, Löwner-Heinz inequality, Furuta inequality.

(C) 1997 American Mathematical Society 


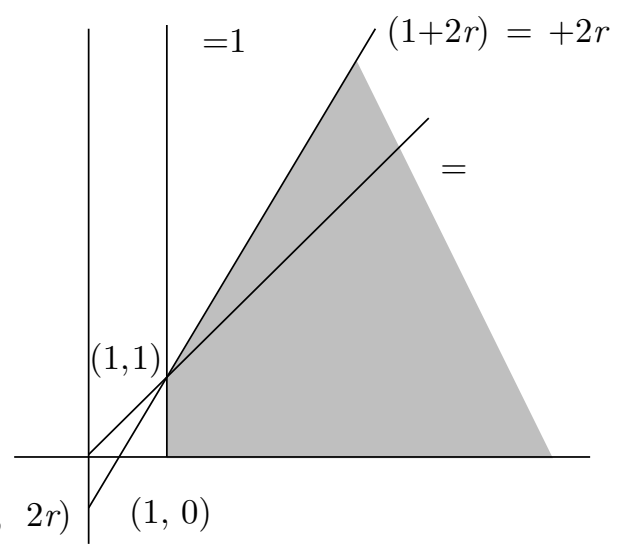

FiguRE 1

Theorem A. For $A, B>0, A \gg B$ if and only if

$$
\left(A^{r} B^{p} A^{r}\right)^{\frac{2 r}{p+2 r}} \leq A^{2 r}
$$

holds for all $p, r \geq 0$.

In this note, we give a simple characterization of the chaotic order. Precisely, $\log A>\log B$ if and only if there is an $\alpha>0$ such that

$$
A^{\alpha}>B^{\alpha} \text {. }
$$

As an application, we can obtain Furuta's type operator inequality implying Theorem A.

\section{Characterization of Chaotic order}

We begin by stating a simple lemma which is the heart of this note:

Lemma 1. If $A$ and $B$ are selfadjoint and $A>B$, then there exists an $\alpha \in(0,1]$ such that

$$
e^{\alpha A}>e^{\alpha B}
$$

Proof. The assumption $A>B$ means that $A-B \geq \varepsilon>0$ for some $\varepsilon$. We here take $0<\alpha<\varepsilon /\left(e^{\|A\|}+e^{\|B\|}\right)$ and $\alpha \leq 1$. Then we have

$$
\begin{aligned}
e^{\alpha A}-e^{\alpha B} & =\alpha(A-B)+\sum_{n=2}^{\infty} \frac{\alpha^{n}}{n !}\left(A^{n}-B^{n}\right) \\
& \geq \alpha \varepsilon+\alpha^{2} \sum_{n=2}^{\infty} \frac{\alpha^{n-2}}{n !}\left(A^{n}-B^{n}\right) \\
& \geq \alpha \varepsilon-\alpha^{2}\left\|\sum_{n=2}^{\infty} \frac{\alpha^{n-2}}{n !}\left(A^{n}-B^{n}\right)\right\| \\
& \geq \alpha \varepsilon-\alpha^{2} \sum_{n=2}^{\infty} \frac{1}{n !}\left(\|A\|^{n}+\|B\|^{n}\right) \\
& \geq \alpha\left(\varepsilon-\alpha\left(e^{\|A\|}+e^{\|B\|}\right)\right)>0 .
\end{aligned}
$$


Lemma 1 implies the following basic inequality:

Corollary 2. If $A, B>0$, then $\log A>\log B$ if and only if there exists an $\alpha \in(0,1]$ such that $A^{\alpha}>B^{\alpha}$.

Proof. If $\log A>\log B$, then $A^{\alpha}>B^{\alpha}$ for some $\alpha \in(0,1]$ by Lemma 1. Conversely, if $A^{\alpha}>B^{\alpha}$ for some $\alpha \in(0,1]$, then $A^{\alpha} \geq B^{\alpha}+\delta$ for some $\delta>0$ and

$$
\alpha \log A=\log A^{\alpha} \geq \log \left(B^{\alpha}+\delta\right)>\log B^{\alpha}=\alpha \log B .
$$

By the above discussion, we have the following simple characterization of the chaotic order:

Theorem 3. For $A, B>0, A \gg B$, i.e., $\log A \geq \log B$, if and only if for any $\delta \in(0,1]$ there exists an $\alpha=\alpha_{\delta}>0$ such that

$$
\left(e^{\delta} A\right)^{\alpha}>B^{\alpha} \text {. }
$$

Proof. Since $A \gg B$ is equivalent to $\log e^{\delta} A=\log A+\delta>\log B$ for any $\delta>0$, Corollary 2 implies that $A \gg B$ is equivalent to saying that for any $\delta>0$ there exists an $\alpha=\alpha_{\delta} \in(0,1]$ such that $\left(e^{\delta} A\right)^{\alpha}>B^{\alpha}$.

We comment that some inequalities related to the chaotic order can be obtained from our result. Among others, we here discuss the Furuta inequality under the chaotic order. Combining Theorem 3 and the Furuta inequality, we have the following lemma:

Lemma 4. If $A, B>0$ and $A \gg B$, then for any $\delta>0$ there exists an $\alpha=\alpha_{\delta} \in$ $(0,1]$ such that

$$
\left(A^{r} B^{p} A^{r}\right)^{\frac{1}{q}} \leq e^{\frac{\delta p}{q}} A^{\frac{p+2 r}{q}}
$$

holds for $p \geq 0, r \geq 0$ and $q \geq 1$ with $(\alpha+2 r) q \geq p+2 r$.

Thus we have the following result equivalent to Theorem A:

Theorem 5. If $A, B>0$ and $A \gg B$, then

$$
\left(A^{r} B^{p} A^{r}\right)^{\frac{1}{q}} \leq A^{\frac{p+2 r}{q}}
$$

holds for $p \geq 0, r \geq 0$ and $q \geq 1$ with $2 r q \geq p+2 r$.

The point of the proof is that if $p, q$ and $r$ satisfy the above condition, then $(\alpha+2 r) q \geq p+2 r$ for all $\alpha>0$.

\section{REFERENCES}

1. T.Ando, On some operator inequalities, Math. Ann., 279 (1987), 157-159. MR 89c:47019

2. M.Fujii, Furuta's inequality and its mean theoretic approach, J. Operator theory, 23 (1990), 67-72. MR 91g:47012

3. M.Fujii,T.Furuta and E.Kamei, Operator functions associated with Furuta's inequality, Linear Alg. and its Appl., 179 (1993), 161-169. MR 93j:47026

4. M.Fujii and E.Kamei, Furuta's inequality and a generalization of Ando's Theorem, Proc. Amer. Math. Soc., 115 (1992), 409-413. MR 92i:47018

5. T.Furuta, $A \geq B \geq 0$ assures $\left(B^{r} A^{p} B^{r}\right)^{1 / q} \geq B^{(p+2 r) / q}$ for $r \geq 0, p \geq 0, q \geq 1$ with $(1+2 r) q \geq p+2 r$, Proc. Amer. Math. Soc., 101 (1987), 85-88. MR 89b:47028

6. T.Furuta, Elementary proof of an order preserving inequality, Proc. Japan Acad., 65 (1989), 126. MR 90g: 47029 
7. E.Kamei, A satellite to Furuta's inequality, Math. Japon., 33 (1988), 883-886. MR 89m:47011

8. G.K.Pedersen, Some operator monotone functions, Proc. Amer. Math. Soc., 36 (1972), 309310. MR 46:6078

Department of Mathematics, Osaka Kyoiku University, Asahigaoka, Kashiwara, OsAKA 582 , JAPAN

E-mail address: mfujii@cc.osaka-kyoiku.ac.jp

Department of Mathematics, Osaka Kyolku University, Kashiwara, Osaka 582, Japan

Permanent address: Department of Basic Science and Technology, China Textile University, Shanghai, China, Postal code 200051

Momodani Senior High School, Ikuno, Osaka 544, Japan

Current address: Maebashi Institute of Technology, Kamisadori, Maebashi, Gunma 371, Japan

E-mail address: kamei@maebashi-it.ac.jp 